\title{
Urgent Thoracotomy Operations In COVID-19 Era
}

\author{
Baris Hekimoglu ${ }^{1(\mathrm{ID})}$,Muhammet Ali Beyoglu ${ }^{2(\mathrm{ID}),}$ Mehmet Furkan Sahin ${ }^{2(\mathrm{ID})}$ \\ ${ }^{1}$ Department of Thoracic, Surgery Faculty of Medicine, Ordu University, Ordu, Turkey, \\ ${ }^{2}$ Department of Thoracic, Surgery Faculty of Medicine, Ankara City Hospital, Ankara, Turkey,
}

Copyright@ Author(s) - Available online at https://dergipark.org.tr/en/pub/mbsjohs Content of this journal is licensed under a Creative Commons Attribution-NonCommercial 4.0 International License,

Received: 15 Ağustos August 2021, Accepted: 16 October 2021, Published online: 31 December 2021 (C) Ordu University Institute of Health Sciences, Turkey, 2021

\begin{abstract}
Objective: This study has aimed to share the perioperative management practices and respective outcomes in patients; who underwent urgent thoracotomy due to traumatic hemothorax during the pandemic.

Methods: In a single-center, 18 patients; who underwent urgent thoracotomy due to traumatic hemothorax in March 2020 to March 2021, were included in the study retrospectively. Patient data were retrieved from digital archive files. The initial evaluation was performed in the emergency room while wearing complete personal protective equipment. Patients were taken into the operating room under emergency conditions without waiting for the results of the nucleic acid tests performed on oropharyngeal and nasopharyngeal swabs. The operation was carried out by involving the minimum number of personnel. In the postoperative period, patients were followed up in a negative pressure intensive care room. Isolation measures were maintained until two novel coronavirus nucleic acid tests on oropharyngeal and nasopharyngeal swabs collected 48 hours apart were reported as negative.

Results: During the one-year period in the COVID-19 pandemic, 18 patients were operated on with the indication of urgent thoracotomy. Of the patients, 14 were men (77.8\%), and 4 were women $(22.2 \%)$. Nucleic acid test results were negative in 17 patients (94.5\%). The nucleic acid test result was reported positive in one patient $(5.5 \%)$ for samples taken at the 48th hour. Nucleic acid tests were performed on the oropharyngeal and nasopharyngeal swabs obtained on the fifth and seventh days from the operation personnel. No novel coronavirus transmission occurred in the healthcare personnel. In the postoperative period, 15 patients $(83.3 \%)$ were successfully treated and discharged from the hospital, but 3 patients (16.7\%) died. No morbidity or mortality occurred due to COVID-19.

Conclusion: Urgent thoracotomies can be successfully performed during the COVID-19 pandemic. Novel coronavirus transmissions can be avoided if relevant healthcare personel comply with isolation measures and use complete personnel protective equipment.
\end{abstract}

Keywords: COVID-19, Thoracotomy, Surgery

Suggested Citation: Hekimoglu B, Beyoglu M A, Sahin M F. Urgent Thoracotomy Operations In COVID-19 Era. Mid Blac Sea Journal of Health Sci, 2021; 7(3):340-347.

\section{Address for correspondence/reprints:}

E-mail: drbarishekimoglu@yahoo.com

Barış Hekimoğlu

Telephone number: +90 (452) 2127245 


\section{Introduction}

The coronavirus disease-2019 (COVID-19) pandemic, caused by coronavirus type 2 (SARSCoV-2), was declared a pandemic by the World Health Organization (WHO) on 11 March 2020. Although it has been 15 months since the declaration, the pandemic continues to challenge healthcare systems globally. According to WHO, 113 million people have been infected with SARS-CoV-2, and 2.5 million of these infected people died as of March 2021 (1). In many countries, healthcare services other than emergency and oncological treatments had to be postponed due to the pandemic. Even the postponement of oncological procedures has been discussed, especially when the density of cases was high $(2,3)$. The Republic of Turkey (T.R.) Ministry of Health directive issued on April 2020 instructed that surgical operations other than emergency and cancer surgery should be postponed in our country (4). Implementing necessary adjustments according to the requirements emerged due to the pandemic, emergency, and oncological surgical interventions of thoracic surgery continued in the period after the directive.

Urgent thoracotomy is the most common non-elective intervention in thoracic surgery. Urgent thoracotomy is a surgical procedure performed in cases where excessive bleeding occurs in the hemithorax following thoracic trauma. Generally accepted urgent thoracotomy indications include the state of shock developing after thoracic trauma, the presence of a major repairable intrathoracic injury, and continuous thoracic hemorrhage (5). Continuous thoracic hemorrhage is defined as the occurrence of hemorrhagic drainage of $>1500 \mathrm{ml}(20 \mathrm{ml} / \mathrm{kg})$ during tube thoracostomy or the drainage of $>250 \mathrm{ml} /$ hour within 3 hours after tube thoracostomy (5). This study has aimed to share the implemented measures and obtained outcomes in patients; who underwent urgent thoracotomy due to traumatic hemothorax during the COVID-19 pandemic.

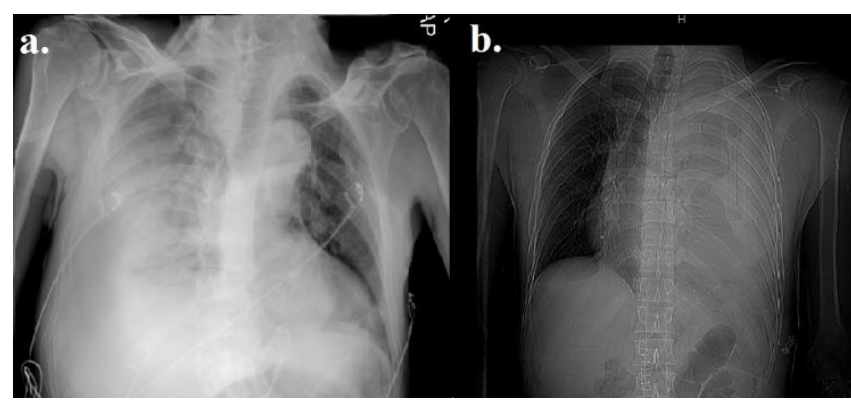

Figure 1. a) Chest radiography of patient 6 reveals right side hemothorax. b) Chest radiography of patient 9 reveals left side hemothorax.

\section{Methods}

This study was designed as a retrospective, descriptive, and cross-sectional study. In the one year between March 2020 and March 2021, patients; who underwent urgent thoracotomy in a secondary stage health center were retrospectively included in the study. A total of 18 patients admitted to the emergency department due to blunt or penetrating chest trauma and on whom urgent thoracotomy and bleeding control were performed due to massive intrathoracic hemorrhage were included in the study. Patient information was obtained from digital and physical archives.

Patients were evaluated in the emergency room in compliance with the T.R. Ministry of Health guideline about the examination of patients suspected of having COVID-19 or diagnosed with COVID-19. Each patient was evaluated by a thoracic surgeon wearing personal protective equipment (PPE). PPE included a bonnet, a face shield, goggles, an N-95 mask, a surgical mask, double gloves, and coveralls or a disposable surgical gown. After taking the anamnesis, performing a physical examination, and laboratory tests including a complete blood count, serum biochemistry, coagulation parameters, blood type tests, direct chest radiography, and thoracic computed tomography (CT) were performed in patients with stable hemodynamics (Figure 1). Patients were inquired not only about the history of the trauma but about COVID-19 as well. Patients were asked whether they had any fever, fatigue, chills, shivering, headache, loss of smell, generalized muscle pain, and diarrhea in the ten days preceding the trauma. Patients were also inquired about whether they visited any places known to be densely populated with COVID-19 cases or whether they had any contact with persons diagnosed with COVID-19 or suspected of having COVID-19 in the last two weeks. Nasopharyngeal (NP) and oropharyngeal (OP) swabs were obtained from patients to perform the SARS-CoV-2 Reverse Transcriptase PolymeraseChain-Reaction (RT-PCR) test. In patients with stable hemodynamics, tube thoracostomy was performed for the treatment of hemothorax. Immediately after the tube thoracostomy, patients with a hemorrhagic drainage volume of $1500 \mathrm{ml}$ or 20 $\mathrm{ml} / \mathrm{kg}$ underwent urgent thoracotomy without waiting for the results of the SARS-CoV-2 RT-PCR test. Anamnesis could not be obtained from patients who had unstable hemodynamics and were in a severe hemorrhagic shock. Such patients underwent surgery without undergoing any radiological imaging tests. Immediately after the operation, an anesthesiologist or a surgeon wearing full PPE took NP and OP swabs 
from patients whose samples could not be taken in the emergency room for the SARS-CoV-2 RT-PCR test.

Urgent thoracotomy was performed through a posterolateral incision under general anesthesia while the patient was lying in the lateral decubitus position. The same surgical team performed all operations. The fewest possible personnel were involved in the operations. A high-efficiency particulate air (HEPA) filter was applied to the draining channel of the thoracostomy drainage bottles before the patients were moved to the intensive care unit (ICU) after the operation. In order to prevent the aerosol generation, all patients were extubated after being transferred to intensive care rooms equipped with negative air pressure. Patients with stable hemodynamics and patients, who did not need mechanical ventilation, were transferred to single rooms in the inpatient unit after 24 hours of intensive care follow-up. All patients were assumed to have suspected COVID-19 until negative results were reported for two consecutive SARS-CoV-2 RT-PCR tests performed 48 hours apart. All interventions were performed by personnel wearing PPE until the suspicion of COVID-19 was ruled out. Patients were followed up through daily postoperative chest radiography images, complete blood counts, and tube thoracostomy drainages. In the presence of fever, leukopenia, and heterogeneous pneumonic infiltration on chest X-rays during the follow-up period, patients underwent nucleic acid tests for the differential diagnosis of COVID-19.

\section{Statistical analysis}

Statistical data were calculated using the SPSS 22.0 software (SPSS Inc., Chicago, IL, USA). Descriptive data were expressed as mean (standard deviation) for the continuous variables and number (\%) for the categorical variables. The Mann-Whitney
$\mathrm{U}$ test was used to determine discrepancies between two classes based on the continuous variables' nonparametric data attributes. A p-value of $<0.05$ was considered statistically significant.

\section{Results}

In the period between March 2020 and March 2021, 18 patients were operated on by the same surgical team in a single-center because of the indication of urgent thoracotomy. Of the patients, 14 were males $(77.8 \%)$, and 4 were females $(22.2 \%)$. The mean age of the patients was 34.9 years (minmax. $=8-77$ years, SD \pm 16.3 ). Descriptive patient information is shown in the Table 1 . Of the patients, $13(72.2 \%)$ reported penetrating thoracic traumas, and $5(27.8 \%)$ reported blunt thoracic traumas in the medical history. Penetrating traumas occurred due to penetrating stab traumas $(n=7 ; 38.9 \%)$, firearm injuries $(\mathrm{n}=3 ; 16.7 \%)$, and foreign body penetrations $(\mathrm{n}=3 ; 16.7 \%)$. Causes of blunt trauma included falling from height $(n=3 ; 16.7 \%)$, falling off a bicycle $(n=1$; $5.5 \%)$, and in-vehicle traffic accidents $(\mathrm{n}=1 ; 5.5 \%)$ (Figure 2)
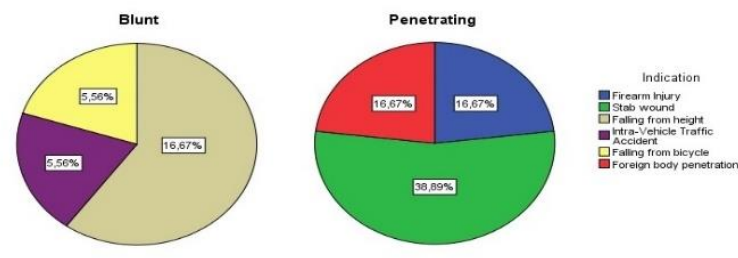

Figure 2: Patient distribution according to the trauma etiology. 
Table 1. Patient demographics and clinical characteristics

\begin{tabular}{|c|c|c|c|c|c|c|c|c|c|c|c|c|}
\hline שֶ & نّّ & $\stackrel{8}{2}$ & 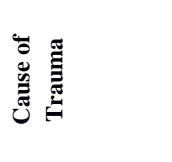 & 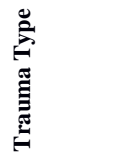 & & 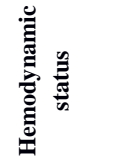 & 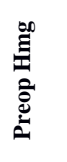 & 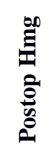 & 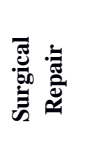 & 苍 & 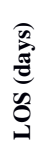 & $\sum_{\tilde{E}}^{n}$ \\
\hline 1 & $\mathrm{M}$ & 40 & Firearm Injury & Penetrating & Right & Stable & 8.0 & $\begin{array}{c}10 . \\
1\end{array}$ & $\begin{array}{c}\text { ICA+ } \\
\text { LP }\end{array}$ & $\mathrm{Neg} / \mathrm{Neg}$ & 22 & $\begin{array}{c}\text { Exitu } \\
\mathrm{s}\end{array}$ \\
\hline 2 & $\mathrm{~F}$ & 30 & $\begin{array}{l}\text { Firearm } \\
\text { Injury }\end{array}$ & Penetrating & Left & Non-stable & 7.3 & 3.6 & $\begin{array}{c}\text { ICA + } \\
\text { Lingula } \\
\text { r Artery }\end{array}$ & $\mathrm{Neg} / \mathrm{Neg}$ & 26 & Alive \\
\hline 3 & M & 50 & $\begin{array}{c}\text { Stab } \\
\text { Wound }\end{array}$ & Penetrating & Left & Stable & 10.6 & $\begin{array}{c}10 . \\
9\end{array}$ & $\begin{array}{c}\text { LIM } \\
\mathrm{A}\end{array}$ & $\mathrm{Neg} / \mathrm{Neg}$ & 13 & Alive \\
\hline 4 & M & 22 & $\begin{array}{c}\text { Foreign Body } \\
\text { Penetration }\end{array}$ & Penetrating & Right & Non-stable & 4.5 & $\begin{array}{c}12 . \\
1\end{array}$ & $\begin{array}{c}\mathrm{ICA}+ \\
\mathrm{LP}\end{array}$ & $\mathrm{Neg} / \mathrm{Neg}$ & 5 & Alive \\
\hline 5 & M & 38 & $\begin{array}{c}\text { Stab } \\
\text { Wound }\end{array}$ & Penetrating & Left & Stable & 11.7 & $\begin{array}{c}11 . \\
0\end{array}$ & ICA & Neg/Neg & 3 & Alive \\
\hline 6 & M & 77 & $\begin{array}{c}\text { Falling from } \\
\text { height }\end{array}$ & Blunt & Right & Stable & 7.6 & 9.2 & $\begin{array}{l}\text { ICA } \\
+\mathrm{LP}\end{array}$ & $\mathrm{Neg} / \mathrm{Neg}$ & 6 & Alive \\
\hline 7 & M & 35 & $\begin{array}{l}\text { Foreign Body } \\
\text { Penetration }\end{array}$ & Penetrating & Left & Stable & 12.1 & $\begin{array}{c}13 . \\
4\end{array}$ & $\begin{array}{c}\text { LP+ } \\
\text { Diap } \\
\text { hrag } \\
\text { ma } \\
\text { Arter }\end{array}$ & $\mathrm{Neg} / \mathrm{Neg}$ & 7 & Alive \\
\hline 8 & M & 59 & $\begin{array}{c}\text { Falling from } \\
\text { height }\end{array}$ & Blunt & Right & Stable & 6.1 & 8.2 & $\begin{array}{c}\mathrm{y} \\
\mathrm{LP}\end{array}$ & $\mathrm{Neg} / \mathrm{Neg}$ & 58 & $\begin{array}{c}\text { Exitu } \\
\mathrm{s}\end{array}$ \\
\hline 9 & M & 45 & $\begin{array}{c}\text { Stab } \\
\text { Wound }\end{array}$ & Penetrating & Left & Stable & 7.4 & $\begin{array}{c}10 . \\
2\end{array}$ & $\begin{array}{c}\text { LIM } \\
\mathrm{A}+ \\
\text { ICA }\end{array}$ & $\mathrm{Neg} / \mathrm{Neg}$ & 5 & Alive \\
\hline 10 & M & 26 & $\begin{array}{c}\text { Stab } \\
\text { Wound }\end{array}$ & Penetrating & Left & Stable & 8.2 & 9.9 & $\begin{array}{c}\text { LP+ } \\
\text { Diap } \\
\text { hrag } \\
\text { ma } \\
\text { Arter }\end{array}$ & $\mathrm{Neg} / \mathrm{Neg}$ & 4 & Alive \\
\hline 11 & $\mathrm{~F}$ & 32 & $\begin{array}{c}\text { Intra-vehicle } \\
\text { Accident }\end{array}$ & Blunt & Left & Stable & 7.1 & 8.5 & $\begin{array}{c}\mathrm{y} \\
\mathrm{ICA}+ \\
\mathrm{LP}\end{array}$ & $\mathrm{Neg} / \mathrm{Neg}$ & 7 & Alive \\
\hline 12 & $\mathrm{~F}$ & 34 & $\begin{array}{c}\text { Falling from } \\
\text { height }\end{array}$ & Blunt & Right & Non-stable & 6.8 & 9.0 & $\begin{array}{c}\mathrm{ICA}+ \\
\mathrm{LP}\end{array}$ & $\mathrm{Neg} / \mathrm{Neg}$ & 5 & Alive \\
\hline 13 & M & 8 & $\begin{array}{c}\text { Falling from } \\
\text { height }\end{array}$ & Blunt & Right & Non-stable & 8.0 & 9.5 & $\begin{array}{l}\text { ICA+ } \\
\text { L.P }\end{array}$ & Neg/Neg & 8 & Alive \\
\hline 14 & $\mathrm{~F}$ & 11 & $\begin{array}{l}\text { Foreign Body } \\
\text { Penetration }\end{array}$ & Penetrating & Right & Non-stable & 7.1 & $\begin{array}{c}10 . \\
2\end{array}$ & L.P & Neg/Neg & 6 & Alive \\
\hline 15 & M & 26 & $\begin{array}{c}\text { Stab } \\
\text { Wound }\end{array}$ & Penetrating & Left & Stable & 4.5 & 7.0 & $\begin{array}{c}\text { ICA+ } \\
\text { LP + } \\
\text { Diap } \\
\text { hrag } \\
\text { ma } \\
\text { Arter } \\
\text { y }\end{array}$ & $\mathrm{Neg} / \mathrm{Neg}$ & 5 & $\begin{array}{c}\text { Exitu } \\
\mathrm{s}\end{array}$ \\
\hline 16 & M & 40 & $\begin{array}{c}\text { Stab } \\
\text { Wound }\end{array}$ & Penetrating & Right & Stable & 6.5 & $\begin{array}{c}10 . \\
3\end{array}$ & $\begin{array}{c}\text { y } \\
\text { RIM } \\
\text { A }\end{array}$ & $\mathrm{Neg} / \mathrm{Neg}$ & 6 & Alive \\
\hline 17 & M & 31 & $\begin{array}{c}\text { Stab } \\
\text { Wound }\end{array}$ & Penetrating & Right & Stable & 8.4 & $\begin{array}{c}11 . \\
0\end{array}$ & $\begin{array}{c}\mathrm{ICA}+ \\
\mathrm{LP}\end{array}$ & Neg/Pos & 11 & Alive \\
\hline 18 & M & 24 & Fire-arm Injury & Penetrating & Left & Stable & 7.9 & 9.0 & $\begin{array}{l}\text { ICA+ } \\
\text { IPV }\end{array}$ & $\mathrm{Neg} / \mathrm{Neg}$ & 10 & Alive \\
\hline
\end{tabular}

Abbreviations: Preop: Pre-operative, Postop: Post-operative, RT-PCR: Novel Coronavirus Reverse Transcriptase Polymerase-Chain-Reaction Test, LOS: Length Of Stay, M: Male, F: Female, ICA.: Inter Costal Artery, LP: Lung Parenchyma, LIMA: Left Internal Mammarian Artery, RIMA: Right Internal Mammarian Artery, IPV: Inferior Pulmonary Vein, Neg: Negative, Pos: Positive. 
Of the patients, who underwent urgent thoracotomy $(\mathrm{n}=18), 9(50 \%)$ had right hemothorax, and $9(50 \%)$ had left hemothorax. Right posterolateral thoracotomy was performed in 9 patients (50\%); left posterolateral thoracotomy was performed in 9 patients (50\%), and bleeding was controlled. It was found out during the operations that bleeding occurred due to traumas to the following structures, including an intercostal artery in $12(66.6 \%)$ patients, the lung parenchyma in $12(66.6 \%)$ patients, a phrenic artery in $3(16.7 \%)$ patients, the left internal mammary artery in $2(11.1 \%)$ patients, the right internal mammary artery in $1(5.5 \%)$ patient, the inferior pulmonary vein in $1(5.5 \%)$ patient, and the lingular pulmonary artery in $1(5.5 \%)$ patient. During operations, an average of 3.6 units of erythrocyte suspension (minimum-maximum $=2-12, \mathrm{SD} \pm 2.5$ ) and an average of 3.8 units of fresh frozen plasma (minimum-maximum $=2-8, \quad \mathrm{SD} \pm 1.9$ ) were administered to patients for replacement. The mean hemoglobin value was $7.7 \mathrm{~g} / \mathrm{dl}$ in the preoperative complete blood counts (min-max. $=4.5-12.1$, $\mathrm{SD} \pm 2.0$ ), and the mean hemoglobin value was 9.6 $\mathrm{g} / \mathrm{dl} \quad$ (min-max $=3.6-13.4, \quad \mathrm{SD} \pm 2.1) \quad$ in the postoperative complete blood counts. There was a statistically significant difference between the preoperative and postoperative hemoglobin values $(\mathrm{p}=0.013)$.

In the postoperative period, patients were followed up in an intensive care room equipped with negative pressure. Thirteen patients $(72.2 \%)$ were extubated just after they were transferred to the intensive care room, and 2 patients $(11.1 \%)$ were extubated at the postoperative 24th hour because blood pressure stabilization could not be achieved in the early postoperative hours. The remaining 3 patients $(16.7 \%)$ were followed under invasive mechanical ventilation for a mean of 27.3 days. These patients were those with severe preoperative blood loss and needed massive blood transfusions during and after surgery. They were followed up on a mechanical ventilator for a long time because of developing respiratory and metabolic acidosis. Of these 3 patients, $2(11.1 \%)$ died due to bacterial sepsis of unknown origin. The other patient (5.5\%) died due to disseminated intravascular coagulation. 15 patients $(83.3 \%)$ were moved to single rooms in the inpatient unit after 24 hours of intensive care follow-up.

Any condition to raise a suspicion of COVID-19 was not observed in the preoperative anamnesis of 13 patients $(72.2 \%)$ who were conscious and did not have a severe hemorrhagic shock. No suspicious conditions were detected in the anamnesis of 5 patients $(27.8 \%)$, whose medical history could only be obtained in the postoperative period because these patients were confused or lethargic due to a severe hemorrhagic shock and impaired consciousness.

Radiologic examinations such as chest $\mathrm{x}$-ray and computed tomography were performed in 13 patients $(72.2 \%)$ who were hemodynamically stable when they first came to the emergency department, in order to show the severity of the trauma. 5 patients $(27.8 \%)$ who were unstable were taken directly to the operating room. No findings associated with COVID19 were detected in the thoracic CT scans of these patients. Tube thoracostomy was performed on these patients in the emergency department. A mean volume of 1738 milliliters (min-max $=1200-2500 \mathrm{ml}$, $\mathrm{SD} \pm 333$ ) hemorrhagic drainage occurred just after the tube thoracostomy.

Only in 1 patient (5.5\%), the result of the RT-PCR test was positive for the swabs taken for the second time 48 hours after the first samples in the postoperative period. This patient, whose SARSCoV-2 RT-PCR test result was reported as positive, had no history of contact with a COVID-19 patient, and had no symptoms associated with COVID-19 as revealed in the anamnesis that could be taken in the postoperative period. In the thoracic CT images, there were no findings in favor of COVID-19. Favipiravir (2x1600mg, 2x400 mg-4 days) therapy was started, and the patient was transferred to the unit, where COVID-19 patients were followed up. No statistically significant difference was obtained in the comparison of the patient with a positive COVID-19 test with other patients in terms of drain termination, hospital stay, and additional complications. NP and OP swab samples for SARS-CoV-2 RT-PCR tests were obtained from the personnel involved in this patient's operation on the 5th and 7th postoperative days. The test results were reported as negative for all of the involved healthcare personnel.

Patients were followed up for COVID-19 in the postoperative period with daily chest radiography, follow-up of the body temperature, and symptom inquiries. In 3 patients (16.7\%), thoracic CT was performed due to postoperative fever; however, no COVID-19-associated findings were observed in the thoracic CT images. The SARS-CoV-2 RT PCR tests on the OP and NP swabs taken from these patients were resulted as negative. The mean length of hospital stay was calculated as 11.8 days $(\mathrm{SD} \pm 13.0)$, and the mean length of intensive care stay was 5.4 days (min-max: $1-58$ days, $\mathrm{SD} \pm 13.8$ ). In the postoperative period, 2 patients $(11.1 \%)$ died due to bacterial sepsis, and 1 patient $(5.5 \%)$ died due to disseminated intravascular coagulation. 15 patients 
(83.4\%) were discharged without the development of any morbidity.

\section{Discussion}

The COVID-19 pandemic caused the postponement of many elective medical and surgical procedures. While continuing the treatment of COVID-19 patients, measures had to be taken to protect healthcare workers and non-COVID-19 patients treated for other reasons in hospitals. In many countries, hospitals are classified as pandemic and non-pandemic hospitals. In areas where the allocation of pandemic hospitals was not possible, isolation measures have been implemented in the hospital. In the early stages of the outbreak, a retrospective study reported that $41.3 \%$ of patients diagnosed with COVID-19 contracted the disease through in-hospital transmission (6). Concerning the density of COVID19 patients in emergency departments, arrangements have been recommended to protect and treat nonCOVID-19 patients and healthcare workers (7-9).

Major trauma patients requiring emergency interventions emerged as a difficult group to manage in this process. Traumatic hemothorax is one of the leading causes of emergencies of thoracic surgery. Traumatic hemothorax is associated with high mortality rates, but timely interventions can be lifesaving. Traumatic hemothorax has become more complex and critical as a condition requiring emergency interventions during the pandemic period, parallel to protecting healthcare workers from this highly infectious infection. There is not an adequate number of papers about the management and outcomes of chest trauma patients who have severe intrathoracic bleeding and face such a life-threatening condition in the literature. Taking necessary preventive measures with the anticipation that each patient could be COVID-19 positive, we performed the necessary interventions on our patients unhesitatingly when emergency surgery was needed during the pandemic.

SARS-CoV-2 is transmitted through droplets when the individual is in close contact with an infected person and through the aerosol particles in the same indoor environment (10). Since SARSCoV-2 has a limited incubation period and can be transmitted by asymptomatic patients, it is highly contagious. Therefore, it is necessary to treat trauma patients as suspected COVID-19 patients in order to protect both healthcare workers and patients (11). Patients should be evaluated by personnel wearing full PPE. Patients with stable hemodynamics and patients not receiving mechanical ventilation support should wear surgical masks. A HEPA filter should be used in the breathing circuit for patients who are mechanically ventilated. If possible, direct radiography should be taken in the room where the patient is. Patients in the present study were examined in the trauma room in the emergency department by healthcare workers wearing full PPE. The transport of the patients to imaging facilities and the operating room was performed by involving the minimum number of personnel. The SARS-CoV-2 transmission was not detected in the emergency room staff and the surgeons of the cases.

It is recommended that patients diagnosed with COVID-19 or who were suspected of having COVID19 should undergo surgery in operating rooms equipped with negative pressure if possible (11-13). Medical supplies and equipment that may be required should be made available for use in the room in advance so that the door of the room should be kept closed throughout the procedure after the patient is admitted to the room (13). General anesthesia and endotracheal intubation are imperative procedures for major surgical procedures such as urgent thoracotomy. General anesthesia and endotracheal intubation are procedures that generate high rates of aerosol $(12,14)$. The management of these procedures should be carried out by the fewest possible personnel wearing full PPE. The cases in our study were managed by a surgical team wearing full PPE. Entrances and exits to the operating room were restricted, and operations were performed with the minimum number of personnel. The door was kept closed during the operation.

Equipment not used in routine operations, such as N95 masks, goggles, face shields, and overalls, cause discomfort for the surgical team during procedures that will take at least two hours. Therefore, the surgeon can be distracted and try to end the operation in a short time (13). Sweating and an inability to retain clear vision due to fogging on the surfaces of goggles and face shields are the most common causes of discomfort for surgeons. As a result, some steps should be taken to combat the surgical team's propensity to not completely use PPE and to avoid premature procedure termination. Airtightness should be ensured around the nose to prevent the formation of fog on the surfaces of goggles. Anti-fog solutions should be applied to goggles and face shields. The ambient operating room temperature should be kept low to help the surgical teamwork comfortably while wearing full PPE. Previous studies have shown that some viruses can survive in surgical smoke (15). Therefore, it is recommended to use energy devices at low power levels, and the surgical smoke should be evacuated continuously. In the present study, the 
aforementioned precautions were taken to prevent the fog formation, maintain the ambient room temperature, and evacuate the surgical smoke so that the surgical team would work comfortably during operations without compromising the use of complete PPE. The healthcare personnel involved in the operation of the patient, who was reported to have a positive nucleic acid test result in the postoperative period, were followed up and underwent the SARSCoV-2 RT-PCR test on NP-OP swabs on the 5th and 7 th days after the operation of the patient. No transmission of SARS-CoV-2 was seen in any personnel.

Tube thoracostomy with closed underwater drainage is one of the thoracic surgery procedures that should be adjusted to the conditions of the pandemic. Via the closed underwater drainage, the air in the thorax is moved to the indoor environment, mixing with the air in the room. Implementation of various procedures has been recommended to prevent aerosol dispersion into the room air $(16,17)$. The most widely used steps are to apply sodium hypochlorite to the bottle fluid and to install a HEPA filter in the bottle's draining tube. In order to prevent the aerosol generation, a HEPA filter was added to the draining channel of the drainage bottles of the study patients.

Extubation of the patient after the operation is a procedure that causes intense aerosol generation. Patients should be extubated by personnel wearing full PPE. In order to prevent the aerosol spread during the transport of the patients, our study patients were extubated after they were transferred to an intensive care room equipped with negative aspiration. In ICU, the provision of treatment and patient visits were performed by personnel wearing full PPE.

A second NP-OP swab sample was taken from each patient at the 48th hour after surgery, and the SARS-CoV-2 RT-PCR test was performed. The test results were negative in all patients except one. The patient, whose test result was positive, was transferred to the COVID-19 inpatient unit and was discharged on the 10th postoperative day without any complications. Thanks to the precautions taken, no transmission of SARS-CoV-2 occurred in the ICU staff and thoracic surgery personnel involved in the provision of treatment and care to the patient.

\section{Conclusion}

Wearing full PPE and compliance with isolation measures in the emergency department, operating rooms, ICU, inpatient units, imaging facilities, transport activities, and disinfection procedures significantly reduce the risk of SARS-CoV-2 transmission. Urgent thoracotomy, which is one of the leading non-elective surgical interventions that cannot be postponed due to the conditions of the COVID-19 pandemic, can be performed safely and successfully if sufficient precautions are taken. Until proven otherwise, trauma patients should be considered suspected COVID-19 cases. At the same time, trauma patients should be protected from SARS-CoV-2 transmission during treatment and follow-up.

\section{Limitations}

The retrospective nature of the research, the small number of cases included, and only one positive SARS-CoV-2 RT-PCR test result in the postoperative period appear to be limiting factors in completely reflecting the interventions' real protective efficacy as mentioned earlier. There is a need for large-scale prospective studies about surgical procedures performed on patients diagnosed with COVID-19.

Ethics Committee Approval: The study was approved by the decision of Ordu University Clinical Research Ethics Committee dated 03/06/2021 with the decision number 128.

Peer-review: Externally peer-reviewed.

\section{Author Contributions:}

Concept: B H, Design: B H, M A B, Literature search: B H, Data Collection and Processing: B H, M A B Analysis or Interpretation: B H, M A B, M F S Writing: B H, M F S

Conflict of Interest: No conflict of interest was declared by the authors.

Financial Disclosure: The authors declared that this study hasn't received no financial support.

\section{References}

1. World Health Organisation Coronavirus (COVID19) Dashboard. Available from: URL: https:// covid19.who.int/. (Accessed on March-2021).

2. Calabrò L, Peters S, Soria JC, Di Giacomo AM, Barlesi $\mathrm{F}$, Covre $\mathrm{A}$, et al. Challenges in lung cancer therapy during the COVID-19 pandemic. Lancet Respir Med. 2020 Jun;8(6):542-544. doi: 10.1016/S2213-2600(20)30170-3.

3. Liang W, Guan W, Chen R, Wang W, Li J, Xu K, et al. Cancer patients in SARS-CoV-2 infection: a nationwide analysis in China. Lancet Oncol. 2020 Mar;21(3):335-337. doi: 10.1016/S1470-2045(20)30096-6. 
4. Ozturk A, Sener MU, Yilmaz A. Bronchoscopic procedures during COVID-19 pandemic: Experiences in Turkey. J Surg Oncol. 2020;122(6):1020-1026. doi:10.1002/jso.26164

5. Karmy-Jones R, Jurkovich GJ, Nathens AB, Shatz DV, Brundage S, Wall MJ Jr, et al. Timing of urgent thoracotomy for hemorrhage after trauma: a multicenter study. Arch Surg. 2001 May;136(5):513-8. 10.1001/archsurg. 136.5.513.

6. Wang D, Hu B, Hu C, Zhu F, Liu X, Zhang J, et al. Clinical Characteristics of 138 Hospitalized Patients With 2019 Novel Coronavirus-Infected Pneumonia in Wuhan, China. JAMA. 2020 Mar 17;323(11):1061-1069. doi: 10.1001/jama.2020.1585.

7. Garcia-Castrillo L, Petrino R, Leach R, Dodt C, Behringer W, Khoury A, et al. European Society For Emergency Medicine position paper on emergency medical systems' response to COVID19. Eur J Emerg Med. 2020 Jun;27(3):174-177. doi: 10.1097/MEJ.0000000000000701.

8. Schreyer KE, Del Portal DA, King LJL, Blome A, DeAngelis M, Stauffer K, et al. Emergency Department Management of the Covid-19 Pandemic. J Emerg Med. 2020 Dec;59(6):946951. doi: 10.1016/j.jemermed.2020.07.022.

9. Coimbra R, Edwards S, Kurihara H, Bass GA, Balogh ZJ, Tilsed J, et al. European Society of Trauma and Emergency Surgery (ESTES) recommendations for trauma and emergency surgery preparation during times of COVID-19 infection. Eur J Trauma Emerg Surg. 2020 Jun;46(3):505-510. doi: 10.1007/s00068-02001364-7.

10.Lin L, Niu LL, Zheng E, Yuan Y, Ning N, Yang M. Management strategies in a thoracic surgery ward during COVID-19 pandemic: Experience from West China Hospital. World J Virol. 2020;9(4):47-53. doi:10.5501/wjv.v9.i4.47

11.Huda F, Kumar P, Singh SK, Agrawal S, Basu S. Covid-19 and surgery: Challenging issues in the face of new normal - A narrative review. Ann Med Surg (Lond). 2020;60:162-167. doi:10.1016/j.amsu.2020.10.039

12.Miller L, Luković E, Wagener G. Guiding airway management and personal protective equipment for COVID-19 intubation teams. Br J Anaesth. 2020;125(3):e288-e290.

doi:10.1016/j.bja.2020.06.001
13.Jheon S, Ahmed AD, Fang VW, Jung W, Khan AZ, Lee JM, Nakajima Jet al. General thoracic surgery services across Asia during the 2020 COVID-19 pandemic. Asian Cardiovasc Thorac Ann. 2020 Jun;28(5):243-249. doi: 10.1177/0218492320926886.

14.Şentürk M, El Tahan MR, Szegedi LL, Marczin N, Karzai W, Shelley B, et al. Thoracic Anesthesia of Patients With Suspected or Confirmed 2019 Novel Coronavirus Infection: Preliminary Recommendations for Airway Management by the European Association of Cardiothoracic Anaesthesiology Thoracic Subspecialty Committee. J Cardiothorac Vasc Anesth. 2020 Sep;34(9):2315-2327. doi: 10.1053/j.jvca.2020.03.059.

15.Chakrabarti A, Bandyopadhyay M. Understanding the practice of thoracic surgery during the COVID-19 pandemic (published online ahead of print, 2020 Aug 11). Indian J Thorac Cardiovasc Surg. 2020;36(5):1-5. doi:10.1007/s12055-02001025-9

16.Pieracci FM, Burlew CC, Spain D, Livingston DH, Bulger EM, Davis KA, et al. Tube thoracostomy during the COVID-19 pandemic: guidance and recommendations from the AAST Acute Care Surgery and Critical Care Committees. Trauma Surg Acute Care Open. 2020 Apr 30;5(1):e000498. doi: 10.1136/tsaco-2020000498.

17.Ceylan KC, Batihan G, Yazgan S, Gürsoy S, Kıraklı SC, Ataman S. Pleural complications in patients with coronavirus disease 2019 (COVID19): how to safely apply and follow-up with a chest tube during the pandemic?. Eur J Cardiothorac Surg. 2020;58(6):1216-1221. doi:10.1093/ejcts/ezaa356 